We have used in this study a combination of genome expression profiling and cytokine arrays to screen for candidates mediating MSC homing to two different cancer cell lines: A549 and MDAMB231. We found a variety of interleukines and cytokines already described as players in the process, such as IL6, IL8, CCL2. Additionally, from in vitro migration and invasion assays, we show that CXCR4 is a major player in this mechanism being the essential MSC receptor for the process to occur. Furthermore, we have identified MIF as the major trigger for MSC homing, being secreted from tumour cells at high levels.

For the first time, we have identified in this study a novel axis: MIF-CXCR4, showing a physical interaction between them and validating their essential role in vitro and in vivo. Importantly, knocking down the expression of CXCR4 in MSCs or MIF in tumour cells, drastically decreased MSC recruitment to tumours in a in vivo model of lung metastasis.

A better understanding of MSC homing players towards tumours will help the development of novel strategies in their use as vehicles in cancer cell therapy.

\section{S36 DIFFERENTIATION OF TUBERCULOSIS AND SARCOIDOSIS BY TRANSCRIPTIONAL PROFILING OF IMMUNE RESPONSES IN MEDIASTINAL LYMPH NODE SAMPLES}

${ }^{1} \mathrm{GS}$ Tomlinson, ${ }^{1} \mathrm{G}$ Hardavella, ${ }^{1} \mathrm{~B}$ Brown, ${ }^{1} \mathrm{~L}$ Succony, ${ }^{2} \mathrm{~N}$ Navani, ${ }^{1} \mathrm{~N}$ Thomas, ${ }^{1} \mathrm{BM}$ Chain, ${ }^{1} \mathrm{SM}$ Janes, 'M Noursadeghi; 'University College London, London, United Kingdom; ${ }^{2}$ University College London Hospital, London, United Kingdom

\subsection{6/thoraxjnl-2013-204457.43}

Introduction and Objectives Differentiating tuberculosis and sarcoidosis can be difficult, particularly in the context of mediastinal lymphadenopathy, because both diseases are characterised by overlapping clinical phenotypes and histologically similar granulomatous inflammation. Currently, diagnosis relies heavily on microbiological confirmation of tuberculosis which is only available in $<50 \%$ of cases. Therefore, novel diagnostic strategies are needed to prevent morbidity associated with delayed or inappropriate treatment. We tested the hypothesis that genome wide transcriptional profiling of mediastinal lymph node samples obtained by minimally invasive endobronchial ultrasound guidance could identify gene signatures that differentiate tuberculosis and sarcoidosis.

Methods In vivo immune responses were compared in mediastinal lymph node biopsies obtained via endobronchial ultrasound guidance from patients with tuberculosis, sarcoidosis or non-granulomatous disease using genome-wide transcriptional profiling. Machine learning algorithms were used to test the discriminatory power of identified gene signatures which distinguished granulomatous from non-granulomatous disease or tuberculosis from sarcoidosis.

Results Comparison of lymph node genomewide transcriptional profiles by principal component analysis revealed clear differences between granulomatous and non-granulomatous disease. Granulomatous profiles showed significant enrichment for genes involved in antigen presentation, inflammatory responses, innate immune responses and $\mathrm{T}$ cell activation, in keeping with the processes involved in granuloma generation. As expected, sarcoidosis and tuberculosis sample profiles were very similar, however, significant gene expression differences were still evident between these two groups. In particular, several genes related to development of granuloma architecture were more highly expressed in sarcoidosis samples. Next we used machine learning tools in order to test the discriminatory power of differentially expressed gene signatures and found that the support vector machines algorithm correctly classified up to $97 \%$ of granulomatous and non-granulomatous disease cases. Importantly, this technique successfully distinguished sarcoidosis from tuberculosis in up to $100 \%$ cases.

Conclusions Transcriptomic analysis of lymph node samples from the site of disease identifies gene signatures that can reliably distinguish tuberculosis from sarcoidosis using computational classification tools. Our data highlight the superior discriminatory power of multiple gene expression differences over a single marker in complex disease and generate a pathway for biomarker discovery in the management of tuberculosis and sarcoidosis.

\section{S37 MATRIX METALLOPROTEINASES DRIVE COLLAGEN DEGRADATION AND LEUKOCYTE MIGRATION IN HUMAN TUBERCULOSIS: CLINICAL AND CELLULAR STUDIES}

${ }^{1} \mathrm{~T}$ Sathyamoorthy, ${ }^{1} \mathrm{JS}$ Friedland, ${ }^{2} \mathrm{~L}$ Tezera, ${ }^{2} \mathrm{PT}$ Elkington; ' ${ }^{\prime}$ mperial College, London, United Kingdom; ${ }^{2}$ University of Southampton, Southampton, United Kingdom

\subsection{6/thoraxinl-2013-204457.44}

Introduction and Objectives Tuberculosis (TB) causes disease worldwide and multi-drug resistance is rising. Matrix metalloproteinases (MMPs) cause immunopathological lung matrix destruction, which results in transmission, morbidity and mortality. We specifically investigated collagenases in $\mathrm{TB}$, studying secreted MMP-1 and MMP-8, and membrane bound MMP-14.

Methods Plasma was prospectively collected from TB patients $(\mathrm{n}=151)$, respiratory symptomatics $(\mathrm{n}=109)$ and controls $(\mathrm{n}=120)$. Plasma MMP concentrations were measured by Luminex bead array. Induced sputum MMP-14 mRNA from TB patients and controls was quantified by RT-PCR. MMP-14 expression in TB patient biopsies was studied by immunohistochemistry. Human monocytes were stimulated with Mycobacterium tuberculosis (Mtb) $\mathrm{H} 37 \mathrm{Rv}$ or Conditioned Media from Mtb infected monocytes (CoMTb) and MMP-14 measured using flow cytometry. Fluorescent microscopy detected MMP-14 and monocyte driven fluorescent collagen degradation. Monocyte migration was measured by the agarose spot assay.

Results Plasma MMP-1 was elevated in TB (median 229pcg/ml) compared to controls (median $2 \mathrm{pcg} / \mathrm{ml} ; \mathrm{p}<0.001$ ). MMP-8 was increased in TB (median $8359 \mathrm{pcg} / \mathrm{ml}$ ) compared to both respiratory symptomatics (median $3547 \mathrm{pcg} / \mathrm{ml} ; \mathrm{p}<0.001$ ) and controls (median $3236 \mathrm{pcg} / \mathrm{ml} ; \mathrm{p}<0.001$ ), and discriminated TB from respiratory symptomatics with moderate predictive ability, with an area under the curve of 0.79 by receiver operating characteristic analysis. In induced sputum, MMP-14 mRNA (normalised to $\beta$-actin) was increased 3.3-fold in TB compared to controls $(p<0.05)$ and mRNA levels positively correlated with the extent of lung infiltration on chest radiograph $(r=0.483 ; p<0.05)$. Macrophages of TB granulomas in patient biopsies stained strongly positive for MMP14. Mtb increased monocyte MMP-14 surface expression 31.7-fold $(\mathrm{p}<0.05)$ and CoMTb 17.5 -fold $(\mathrm{p}<0.01)$, secondary to increased mRNA. Mtb-infected monocytes degraded collagen, with co-localised MMP-14 surface expression. Monocytes migrated to the edge of CoMTb-impregnated agarose drops, expressing MMP-14 on migration. Inhibition of MMP-14 activity with a neutralising antibody, decreased Mtb driven collagen degradation by $73 \%(\mathrm{p}<$ $0.001)$ and CoMTb driven monocyte migration by $44 \%(\mathrm{p}<0.001)$. Inhibition of chemokine signalling using pertussis toxin reduced CoMTb driven MMP-14 surface expression by 35\% ( $\mathrm{p}<0.05)$.

Discussion Collagen destruction is critical to pathogenesis in pulmonary $\mathrm{TB}$, and these data implicate three collagenases, MMP-1, -8 and -14 , in causing immunopathology and regulating leukocyte migration. MMPs may represent novel diagnostic and therapeutic targets. 